\title{
Processing Verb-phrase Ellipsis through Structural Priming in Mandarin Chinese: Evidence from Mandarin Chinese among Chinese-English Bilinguals
}

\author{
Ellen ZHANG Yue \\ Department of English, Faculty of Arts, The Chinese University of Hong Kong, Hong Kong, China \\ Email: yuezhang@link. cuhk.edu.hk
}

\begin{abstract}
The processing of syntactic and semantic cues while processing structures in learners' foreign language arouse wide concern and discussion. Since equally robust evidence from priming the syntactically and semantically represented accounts occur across studies, how do bilinguals construct a complicated structure including ellipsis using remains to be confirmed, especially when one single author carried out empirical research in the same language and revealed seemingly different results, yielding misunderstanding and calling for further clarification. Using the verb-phrase ellipsis through structural priming in Mandarin Chines, this article aims to critically discuss existing findings with evidence from Mandarin-English bilinguals.
\end{abstract}

Keywords: Verb-phrase ellipsis, structural priming, Chinese-English bilinguals, Mandarin Chinese processing.

\section{Introduction}

In terms of production, bilinguals share the syntactic accounts between two languages with sufficiently similar structures (Hartsuiker et al., 2004), and use separate lemma to represent similar syntactic structures between the two languages they master (Hartsuiker \& Pickering, 2008). For instance, elided verbs mark the pro-drop feature of both Mandarin and English, which indicates that a subject/object of the verb can be omitted with a preceding context provided for people to recover the meaning (Xu, 2003). Wide attention has been directed to the 'NPTheme NPAgent verb-gei-le NPRecipient' structure in Mandarin Chinese (Chang, et al., 2003) as the target structure selected under investigation in this paper. An extra level of complexity is embedded in its comprehension when ellipsis is combined with the double-object dative (DO) and the prepositional-object dative (PO) construction (Bock, 1986) with mixed findings reported (Cai et al., 2011; Cai et al., 2012; Cai et al., 2013; Cai et al., 2015).

Furthermore, although some argue that such ellipsis could be both syntactically and semantically represented (Hankamer, 1979; Merchant, 2001), equally robust support for priming in accordance with the syntactically (Bock \& Loebell, 1990; Cai et al., 2015; Branigan \& Pickering, 2017) and semantically (Chang et al., 2003; Cai et al., 2012; Cai, et al., 2013; Cho- Reyes et al., 2016)represented accounts have been recognized across languages (Ziegler et al., 2017). To fill this void, this paper discusses how Mandarin-English bilinguals construct similar structures in both languages while comprehending sentences with missing arguments from existing literature and tries to expand the scope of discussion. In correspondence to the above-stated underpinning rationale, two questions are raised as follows.

(1). How do Mandarin-English bilinguals construct the DO/PO structures while comprehending sentences with missing arguments?

(2). Moving beyond the existing findings, what could be further explored in processing the DO/PO ellipsis construction?

\section{Experimental Introspection: Structural Priming}

Working in their own linguistic enterprise, linguists study how language is represented and processed in the mind (Lasnik, 2002; Phillips \& Lasnik, 2003; Culicover \& Jackendoff, 2010), which is a focus of psychologists as well. For linguists, judgments are gathered with a minimal pair, which consists of two 
sentences, namely 'an example sentence' which is in lack of grammaticality and the other sentence which shares a certain degree of similarity to the former one (Ferreira, 2005, p.37). Nonetheless, psycholinguists have tended to critically evaluate linguistic evidence because of its lack of empirical precision (Edelman \& Christiansen, 2003; Gibson \& Fedorenko, 2010; 2013).

A case in point is that Hornstein (1995) claimed that the ungrammaticality can be ameliorated if there exists a third wh-element. It has been proved by experimental introspection that there actually is no significant difference in acceptability rating and reaction time between 'What can who do about it?' and 'What can who do about it when?' (Clifton et al., 2006). Nevertheless, this method does not address the issue of plausibility, processibility, and psychological reality, whereas the participants are influenced by them, and to judge them, experimental inference is needed. Taking the vacuous movement sentences as another illustration, on one hand, Chomsky (1986) claimed that the vacuous movement sentences have a higher degree of acceptability than the superiority condition violation sentences. On the other hand, through judgement test on how natural the sentence is, Gibson and Fedorenko (2013) argued that in fact the Superiority Condition violation sentence more acceptable than the vacuous movement sentence.

One way to provide the above-mentioned empirical precision is structural priming, which refers to the tendency for people to repeat aspects of syntax from previous utterances they have produced or comprehended, which can be used as a tool to discriminate different syntactic accounts of a contentious construction (Branigan \& Pickering, 2017). As an illustration, in terms of comprehension, if a Cantonese-Mandarin bilingual reads a cluster of sentence including the DO/PO ellipsis structure in Mandarin, then when he or she is asked to describe a picture, it would be more likely for him or her to produce another sentence with the same structure due to the priming effects. Similarly, regarding production, if the participants are asked to describe a series of pictures first using a particular syntactic structure, then when thery are asked to write an essay, they are expected to repeat it as the target linguistic structure under examination.

In another word, the linguistic representation of a construction is more activated due to prior use with a tendency to use a previous/activated structure to produce a syntactic structure. Within-language and between-language structural priming are separable from semantic and lexical repetition between the prime and the target sentence (Pickering \& Branigan, 1998), and they have been proved to occur in comprehension (Branigan et al., 2005; Traxler, 2008), production (Cai et al., 2011), and between comprehension and production (Branigan et al., 2000; Branigan et al., 2005). Despite of evidence against the omitted categories in relation to the movement of verbs in syntactic constructs, the empty categories that are lack of phonological representation could be syntactically processed (Branigan \& Pickering, 2017).

\section{Processing DP/PO Ellipsis Construction from Three Perspectives}

\subsection{Grammatical or Phonological Encoding}

Answering the first question, in the process of formulation before articulation and after conceptualization, there exist two procedures of encoding (Levelt, 1989), namely the grammatical and the phonological encoding. Using the DO/PO ellipsis construction as an example, how Cantonese-Mandarin bilinguals process the dative alternation to cognate representations depends on whether the cognates in two languages have shared lemma representations and more specifically, which has a larger priming effect (Cai et al., 2011). In two picture-description experiments on the two languages respectively, Cai and his colleagues (2011) have proved to be based on lexico-syntactic representations instead of phonological similarity effects through the priming effects. This supports Hartsuiker and Pickering's argument (2008) that bilinguals retrieve lemmas as lexical representations from both languages to express similar/common syntactic structures between the two languages with separated lexical representations.

\subsection{Functional or Positional Processing}

Under syntactic encoding, there exist three types of processing. Firstly, functional processing denotes the process of choosing a structure and determining the functions of it, like allocating the subject to 'Yige laoshi', the direct object to 'yixie lingshi', and the oblique object to 'xuesheng' in a PO sentence 'Yige 
laoshi songle yixie lingshi gei xuesheng' (One teacher give-ASP some snacks to student.)'. Similarly, it allocates the subject to 'Yige laoshi', the indirect object to 'xuesheng', and the direct object to 'yixie lingshi' in a DO sentence 'Yige laoshi song-gei xuesheng yixie lingshi. (One teacher give-ASP student some snacks.)'. Secondly, the positional processing refers to determining the word order, including sequencing the structures to compose a grammatical sentence, like putting a preposition before an object.

The third processing is concerning morphology, consisting of changing the bare form 'give' to 'gave', 'student' to 'students', and 'snack' to 'snacks' in the sentences, which should be done at the end. With that being said, whether the functional or positional processing comes first remains to be uncovered. Another notion is adopted to examine the sequence, namely the shifted PO, which is the theme shifted to the end of the sentence. For example, in the structure GEI [Yige laoshi, xuesheng, yixie lingshi], a shifted PO is 'yixie lingshi'. If functional processing comes before positional processing, then people should build a functional PO structure first before moving the theme to the end, and if so, a shifted PO should prime/elicit a $\mathrm{PO}$ response. Nevertheless, according to the proportion of $\mathrm{PO}$ responses from high to low, namely ' $\mathrm{PO}>$ Baseline $>$ Shifted-PO $>$ DO', it does not prime due to no significant difference in the proportion of the shifted $\mathrm{PO}$ and the baseline, indicating that the shifted PO is not built on PO (Pickering et al., 2002). In a follow-up study in Mandarin Chinese, the DO '牛仔送给了水手那本书', the PO '牛仔 送了那本书给水手', and the Topic-DO '那本书牛仔送给了水手' were investigated to see whether people first build a DO before transforming it into a Topic-DO, and if so, the Topic-DO primes DO responses. But the results $(\mathrm{DO}>$ Baseline $>$ Topic-PO $>\mathrm{PO})$ indicate that it primes the $\mathrm{PO}$ responses instead of $\mathrm{DO}$ responses, suggesting no immediate stage where people build a DO structure first and then linearize it into a Topic-DO structure. Instead, they build the Topic-DO structure (Cai et al., 2012).

\subsection{Syntactic or Semantic Representations of the Missing Arguments}

The seemingly inconsistent results through structural priming are of a higher level of complexity compared to the grammatical/phonological enquiry. First of all, it resorts to the non-syntactic accounts when the meaning of VP ellipsis is interpreted, provided that people take the antecedent into consideration (Cai et al., 2013) as is in the following sentences in English and Chinese, namely 'One teacher will buy some snacks and then give students some. The other will too.' and 'Yige laoshi mai yixie lingshi hou hui song-gei xuesheng. Lingyige ye hui. (One teacher buy some snacks later give-ASP student some; another also will.)'. However on second thoughts, it does turn to the syntactic accounts as participants do construct the full-form sentences as the constituent structure for both missing-argument and full-form sentences due to the priming effects of a dative sentence plus a missing direct-object argument (Cai et al., 2015).

In view of whether syntactic or semantic account of the ellipsis is supported through structural priming, Cai and his colleagues (2013) examined the VP ellipsis licensed by the modal auxiliary 'xiang' by comparing and contrasting participants' primed responses towards the DO and PO dative structures through 60 ppts, 36 targets and 108 fillers. Although two DOs condition normally has a larger priming effect than the one DO condition, it remains to be proved that whether ellipsis primes to a larger extent on the full or control reveals; whether ellipsis contains an unpronounced semantic structure; and whether the missing argument has a semantic account. To testify such hypotheses, a prime was designed to embody a PO or DO clause followed by a clause with a VP ellipsis to Mandarin speakers. Through examining their descriptions for pictures about dative events, it is revealed that the semantic account of VP ellipsis is supported, whereas no unpronounced syntactic structure at the ellipsis is found.

It is the contrary case when it comes to the missing element being a single argument instead of an entire VP. Another study conducted by the author and his colleagues (Cai, et al., 2015) investigated whether people construct the same nature of syntactic representations for the missing DO and PO sentences like the full forms after comprehending sentences including omitted argument. In the structure of GIVE (MANAGENT, CHARITYRECIPIENT, MONEYTHEME), whether people reconstruct the missing argument in syntax to fill in the blank of 'A charity was in need of funding. The man gave some money 'remains to be uncovered. The DO is '牛 仔 买了一本书后送给了水手那本书。' and the PO is “牛 仔买了一本书后送了那本书给水手。' The DO-MA is '牛仔买了一本书后送给了水手_。' and the baseline is “板球选手在打喷德。' If participants support the syntactic account, then DO-MA = should behave like DO in priming, where as if they support the semantic one, it should be 'DO- MA = Baseline $<$ DO' in the priming effects. The results from the first experiment support the syntactic account that the missing 
argument is reconstructed during comprehension using 48 PowerPoint slides, 32 target sets of materials and 96 filler sets. In the second experiment, the DO, PO and Baseline are the same but the PO=MA becomes '牛仔买了一本书后送了—给水手。' Through Bayes factor analysis, PO = PO-MA is 27 times more likely than $\mathrm{PO} \neq \mathrm{PO}-\mathrm{MA}$, indicating that missing arguments are syntactically represented. Hence, both experiments showed that missing arguments are syntactically represented/ reconstructed in comprehension.

In a nutshell, the results of the two studies (Cai et al., 2013; Cai et al., 2015) show that whether missing arguments are syntactically or semantic represented depends on the missing element being a single argument or an entire VP. To what extent can this be generalized to other languages other than Mandarin remains a mystery owing to the fact that inconsistency exists in English (Raffray et al., 2014) and German (Pappert \& Pechmann, 2013). One possible interpretation of the mixed findings could be that in some languages, $\mathrm{PO} / \mathrm{DO}$ sentences share similar syntactical representation as well as semantic ones, whereas in others, the benefactives carry out different actions with respect to different events that the structures denote (Shibatani, 1996). From the multilingual perspective, arguing whether it accounts for syntactic or semantic representations alone seems to be of less theoretical implications to better understand the processing procedure, hence leading to the following further discussion with practical implications.

\section{Discussion and Practical Implications}

\subsection{Structural Priming Being Fluid and Dynamic}

With regard to the second question, to move beyond the four perspectives discussed above, theoretical implications are addressed as follows. Priming, first of all, should be viewed as fluid and dynamic for representations considered as unstable forms in relation to the process of comprehension and production (Ziegler et al., 2017), as the stability of linguistic representations might affect the validity of structural priming in that priming can change the accounts being investigated. It has been proved feasible to use structural priming in mapping out the developmental trajectories for syntactic constructions (McDonough\& Mackey, 2006), picturing the process of comprehension and production (VanPatten, 2014), revealing the process of learners' comparing and contrasting (Chen et al., 2013), identifying (Clahsen \& Felser, 2006) and representing L1-L2 representations (Cai et al., 2012), and the sequential development (Rowland et al., 2012) from a microscopic approach (Cai \& Liu, 2017). Nonetheless, similar to the results discussed above, it is pointed out that such effects in production could be due to the elicitation of sentences, which makes it vital to better understand such instability (Ziegler et al., 2017).

Moreover, although there exist similar structural priming effects between within- language and between-language priming and also between two L2s and L1-L2 priming (Hartsuiker et al., 2016), the effects of language similarity has been isolated from the procedure. Different monostratal features posit an asymmetry between two expressions of the same idea as follows. English speakers may produce 'I love apples', whereas the sequence of the Italians' utterance is 'apples like. PLUR to I', causing problems for speakers of languages with different monostratal features, and these problems could be detected using structural priming. Therefore, due to the monostratal feature of syntactic representations that embodies the hierarchical relations being the similar features between Mandarin and English, it is reasonable to predict differences in structural priming effects between within-language (Mandarin and English separately) and between-language (Mandarin and English) priming.

Apart from revealing the L1-L2 relationship, structural priming can as well be conducted to examine the extent to which an L2 representation can be identified with its corresponding representation in the target language. It has been claimed that second language learners are in lack of fully fledged syntactic representations (Clahsen \& Felser 2006). However, for Mandarin Chinese, a dative sentence with a missing argument behaves similarly to its corresponding full-form sentence in priming dative sentences, suggesting that native speakers of Mandarin syntactically represent the missing argument even though it is not phonologically realised (Cai et al., 2012). Besides, structural priming can be used to map out the developmental trajectory for syntactic constructions (McDonough \& Mackey 2006). 


\subsection{The Monostratal Feature of DO/PO Construction}

Secondly, the monostratal feature of syntactic representations embodies the hierarchical relations, which lead to failure of priming effects on sentences in English (Pickering et al., 2002) and German (Pappert \& Pechmann, 2013). Drawing from such evidence, it is as well notable to investigate whether participants also have a reduced tendency to produce a PO in the V NP PP order like 'Yige laoshi mai yixie lingshi hou hui song-gei xuesheng. Lingyige ye hui. (One teacher buy some snacks later give-ASP student some; another also will.)', following a changed PO in the V PP NP order like 'Yige laoshi gei xuesheng song yixie lingshi. Lingyige ye hui. (One teacher to student give-ASP some snacks; another also will)'. With that being said, more attention could be directed to the monostratal feature of DO/PO sentences with VP ellipsis in Mandarin processing, alongside some given to the Mandarin as second language learners.

\subsection{Participants' Background}

Considering participants' demographic information, it is worth noticing that more information about the participants are needed to compare and contrast the results accordingly. For instance, the participants in the former study are sixty 'participants from universities in Guangzhou, China' (Cai et al., 2013, p. 816), while those in the latter are 'forty-eight native speakers of Mandarin Chinese from the South China Normal University Community' (Cai et al., 2015, p. 260). Given more word counts, detailed background and demographic information of the participants like the age, gender, linguistic background and other information could also be provided to enhance the validity and reliability of the experiments.

In fact, gender, age, self-ratings of proficiency level, left-or- right-handedness, the year of becoming literate in Mandarin before learning English, and educational background might work as possible influential factors on the priming effects (Huang et al., 2016; Hartsuiker et al., 2016; Bulkes \& Tanner, 2018). That is to say, between two L2s (English and German as the second languages with dative alternation being etymologically similar to Mandarin) and L1 (Mandarin)-L2 (English) priming, proficiency level of the language is also taken off in the experiments (Cai et al., 2013; Cai, et al., 2015), which could be a factor to be examined in a follow-up study to check whether it influences the differences significantly.

Based on both within-language and between-language priming for Cantonese and Mandarin, CantoneseMandarin bilinguals are revealed to possess different lemmas, differentiating at the lexico-syntactic level (Cai et al., 2011). Japanese and Korean Chinese as a Foreign Language (CFL) learners used semantic and phonetic radicals equally, while the English group preferred semantic radicals (Tong et al., 2016). Therefore, whether CFL learners also differ in their processing procedure and performance remains to be revealed.

\subsection{Pedagogical Implications}

Last but not least, pedagogically speaking, being an L2 construction that co-opts the L1 structure, the DO/PO sentences with VP ellipsis sometimes account for L1 transfer in L2 among Hong Kong Chinese ESL learners (Chan, 2004) and for varieties like Hong Kong English (Ting et al., 2018). It has been further proved that for elementary school students, the multimodal presentation of stories in English enhance retelling performance in Chinese (Chan et al., 2019). Beyond the Chinese context, such transfer effects also lead to errors at undergraduate level in learners' production in writing (Burhansyah, 2019), reading (Choi et al., 2018) and spelling achievement (Gibree et al., 2018), but could be corrected to improve students' accuracy through strategies like peer-teacher feedback (Nguyen, 2018).

\section{References}

1. Bock, J. K. (1986). Syntactic persistence in language production. Cognitive Psychology, 18(3), 355-387.

2. Bock, K. \& Loebell, H. (1990). Framing sentences. Cognition, 35(1):1-39.

3. Branigan, H. P., \& Pickering, M. J. (2017). An experimental approach to linguistic representation. Behavioral and Brain Sciences, 40(1). 1-61.

4. Branigan, H. P., Pickering, M. J., \& Cleland, A. A. (2000). Syntactic coordination in dialogue. Cognition, 75(2), B13-B25. 
5. Branigan, H. P., Pickering, M. J., \& McLean, J. F. (2005). Priming prepositional-phrase attachment during language comprehension. Journal of Experimental Psychology: Learning, Memory, and Cognition, 31(3), 468-481.

6. Branigan, H., \& Pickering, M. (2017c). An experimental approach to linguistic representation. Behavioral and Brain Sciences, 40.(1), 1-61.

7. Bulkes, N., \& Tanner, D. (2018). Quantificational cues modulate the processing of English subject-verb agreement by native Chinese speakers: An ERP study. Studies in Second Language Acquisition, 40(4), 731-754.

8. Cai, Z. G., \& Liu, H. (2017). Microscopic and macroscopic approaches to the mental representations of second languages. Behavioural and Brain Sciences, 40, 19-20 [commentary].

9. Cai, Z. G., Pickering, M. J., \& Branigan, H. P. (2012). Mapping concepts to syntax: Evidence from structural priming in Mandarin. Journal of Memory and Language, 66(4), 833-849.

10. Cai, Z. G., Pickering, M. J., \& Sturt, P. (2013). Processing verb-phrase ellipsis in Mandarin Chinese: Evidence against the syntactic account. Language and Cognitive Processes, 28(6), 810-828.

11. Cai, Z. G., Pickering, M. J., Wang, R., \& Branigan, H. P. (2015). It is there whether you hear it or not: Syntactic representation of missing arguments. Cognition, 136, 255- 267.

12. Cai, Z. G., Pickering, M. J., Yan, H., \& Branigan, H. P. (2011). Lexical and syntactic representations in closely related languages: Evidence from Mandarin and Cantonese. Journal of Memory and Language, 65(4), 431-445.

13. Chan, A. Y. (2004). Syntactic transfer: Evidence from the interlanguage of Hong Kong Chinese ESL learners. Modern Language Journal, 88(1), 56-74.

14. Chang, F., Bock, J. K., \& Goldberg, A. (2003). Can thematic roles leave traces of their places? Cognition, 90(1), 29-49.

15. Chomsky, N. (1986). Knowledge of language: Its nature, origin, and use. Greenwood Publishing Group.

16. Cho-Reyes, S., Mack, J. E. \& Thompson, C. K. (2016). Grammatical encoding and learning in agrammatic aphasia: Evidence from structural priming. Journal of Memory and Language, 91, 202-18.

17. Clifton Jr, C., Fanselow, G., \& Frazier, L. (2006). Amnestying superiority violations: Processing multiple questions. Linguistic Inquiry, 37(1), 51-68.

18. Culicover, P. W., \& Jackendoff, R. (2010). Quantitative methods alone are not enough: Response to Gibson and Fedorenko. Trends in Cognitive Sciences, 14(6), 234-235.

19. Dubinsky, S., Egan, M., Schmauder, A. R., \& Traxler, M. J. (2000). Functional projections of predicates: experimental evidence from coordinate structure processing. Syntax, 3(3), 182-214.

20. Edelman, S., \& Christiansen, M. H. (2003). How seriously should we take minimalist syntax? A comment on Lasnik. Trends in Cognitive Science, 7(2), 60-61.

21. Ferreira, M. M. B. (2005). Event quantification and plurality (Doctoral dissertation, Massachusetts Institute of Technology).

22. Gibson, E., \& Fedorenko, E. (2010). Weak quantitative standards in linguistics research. Trends in cognitive sciences, 14(6), 233-234.

23. Gibson, E., \& Fedorenko, E. (2013). The need for quantitative methods in syntax and semantics research. Language and Cognitive Processes, 28(1-2), 88-124.

24. Hankamer, J. (1971). Deletion in Coordinate Structures, unpublished Ph. D (Doctoral dissertation, dissertation, Yale University).

25. Hartsuiker, R. J., Beerts, S., Loncke, M., Desmet, T., \& Bernolet, S. (2016). Cross- linguistic structural priming in multilinguals: Further evidence for shared syntax. Journal of Memory and Language, 90, 14-30.

26. Hartsuiker, R. J., Pickering, M. J., \& Veltkamp, E. (2004). Is syntax separate or shared between languages? Cross-linguistic syntactic priming in Spanish-English bilinguals. Psychological Science, 15, 409-414.

27. Hornstein, N. (1995). Logical form: From GB to minimalism. Blackwell.

28. Huang, J., Pickering, M. J., Yang, J., Wang, S., \& Branigan, H. P. (2016). The independence of syntactic processing in Mandarin: Evidence from structural priming. Journal of Memory and Language, 91, 81-98.

29. Lasnik, H. (2002). The minimalist program in syntax. Trends in cognitive sciences, 6(10), 432-437.

30. McDonough, K., \& Mackey, A. (2006). Responses to recasts: Repetitions, primed production, and linguistic development. Language Learning, 56(4), 693-720.

31. Merchant, J. (2001). The syntax of silence: Sluicing, islands and the theory of ellipsis. Oxford University Press on Demand.

32. Pappert, S., \& Pechmann, T. (2013). Bidirectional structural priming across alternations: Evidence from the generation of dative and benefactive alternation structures in German. Language and Cognitive Processes, 28(9), 1303-1322. 
33. Phillips, C., \& Lasnik, H. (2003). Linguistics and empirical evidence: Reply to Edelman and Christiansen. Trends in cognitive sciences, 7(2), 61-62.

34. Pickering, M. J., \& Branigan, H. P. (1998). The representation of verbs: Evidence from syntactic priming in language production. Journal of Memory and Language, 39,633-651.

35. Pickering, M. J., Branigan, H. P., \& McLean, J. F. (2002). Constituent structure is formulated in one stage. Journal of Memory and Language, 46, 586-605.

36. Raffray, C. N., Pickering, M. J., Cai, Z. G., \& Branigan, H. P. (2014). The production of coerced expressions: Evidence from priming. Journal of Memory and Language, 74, 91-106.

37. Shibatani, M. (1996). Applicatives and benefactives: A cognitive account. In Shibatani, M., \& Thompson, S. A. (Eds.). (1999). Grammatical constructions: their form and meaning (pp. 157-194). Oxford University Press, USA.

38. Tong, X., Kwan, J. L. Y., Wong, D. W. M., Lee, S. M. K., \& Yip, J. H. Y. (2016). Toward a dynamic interactive model of non-native Chinese character processing. Journal of Educational Psychology, 108(5), 680-693.

39. Traxler, M. J. (2008). Lexically independent priming in online sentence comprehension. Psychonomic Bulletin 83 Review, 15 (1), 149-155.

40. Wei, H., Boland, J., Cai, Z., Yuan, F., Wang, M., \& Benjamin, Aaron S. (2019). Persistent Structural Priming During Online Second-Language Comprehension. Journal of Experimental Psychology: Learning, Memory, and Cognition, 45(2), 349-359.

41. Xu, L. J. (2003). Remarks on VP-ellipsis in disguise. Linguistic Inquiry, 34, 163-171.

42. Ziegler, J., Snedeker, J., \& Wittenberg, E. (2017). Priming is swell, but it's far from simple. Comments on Branigan \& Pickering (2017). Brain and Behavioral Sciences, 40, e312. 